\title{
Right Superior Temporal Gyrus Altered Functional Activity in Patients With Definite Vestibular Migraine
}

\section{Xu Yang ( $\nabla$ yangxu2011@163.com )}

Peking university Aerospace school of clinical medicine https://orcid.org/0000-0002-3615-3063

Zhe-Yuan Li

Peking university aerospace school of medicine

Li-Hong Si

Peking university aerospace school of clinical medical

Bo Shen

Peking university aerospace school of clinical medicine

Xia Ling

Peking university aerospace school of clinical medicine

\section{Research Article}

Keywords: vestibular migraine, spontaneous brain activity, functional connectivity, ALFF, fALFF, ReHo

Posted Date: January 14th, 2022

DOI: https://doi.org/10.21203/rs.3.rs-1206351/v1

License: () (i) This work is licensed under a Creative Commons Attribution 4.0 International License. Read Full License 


\section{Abstract}

The study aimed to investigate resting-state functional brain activity alterations in patients with definite vestibular migraine (dVM). Seventeen patients with dVM, 8 patients with migraine, 17 health controls (HCs) were recruited. The amplitude of low frequency fluctuation (ALFF), fractional ALFF (fALFF) and regional homogeneity (ReHo) were calculated to observe the changes in spontaneous brain activity. Then brain regions with altered fALFF were selected for seed-based functional connectivity analysis. Compared with HCs, VM patients showed significantly increased ALFF values in the right temporal lobe (Cluster size $=91$ voxels, $P=0.002$, FWE corrected), and significantly increased ReHo values in the right superior temporal gyrus (STG), middle temporal gyrus (MTG) and inferior temporal gyrus (ITG) (Cluster size $=136$ voxels, $P=0.013$, FWE corrected). Compared with patients with migraine, patients with VM showed significantly increased fALFF values in the right parietal lobe (Cluster size $=43$ voxels, $P=0.011$, FWE corrected) and right frontal lobe (Cluster size $=36$ voxels, $P=0.026$, FWE corrected), significantly increased ReHo values in the right thalamus (Cluster size $=92$ voxels, $\mathrm{P}=0.043$, FWE corrected). Our findings documented that patients with VM showed enhanced spontaneous functional activity in the right temporal lobe (STG, MTG, and ITG) compared with HCs, and increased spontaneous activity in the right parietal lobe-frontal lobethalamus compared with patients with migraine. Patients with VM and migraine both had altered brain function, but the regions involved are different.

\section{Introduction}

Vestibular migraine (VM) is considered the most common cause of central episodic vertigo, accounting for $7 \%$ of patients seen in neurootological clinics and $9 \%$ of patients seen in headache clinics (Dieterich et al. 2016). In 2012, diagnostic criteria for VM was jointly formulated by the Bárány Society and the Migraine Classification Subcommittee of the International Headache Society, which were published in the International Classification of Headache Disorders, 3rd edition (beta version) (ICHD-III beta) in 2013 (Headache Classification Committee 2013). At present, the clinical diagnosis of VM relies on clinical symptoms (Lempert et al. 2012). During VM attacks, patients usually have abnormalities in perception of movement, which are often accompanied by the sensation of rotation or sway, increased visual motion sensitivity and spatial disorientation (Agarwal et al. 2012). During the interictal period, nystagmus may be observed in patients, suggesting that there is a certain degree of brain stem involvement (Polensek and Tusa 2010). Among the hypotheses about the mechanisms of VM, most hypotheses categorize VM as a subcategory of migraine. However, the current vestibular testing techniques alone are not yet able to make the differentiation of VM from some vestibular diseases (von Brevern et al. 2005). Some researches suggest peripheral damage in VM patients. In these cases, opinions diverge as to whether peripheral damage in a migraine subtype is possible, or must then be a different entity such as a monotypical Menière's disease in VM patients with endolymphatic hydrops (Balaban 2011; Furman et al. 2013; Pyykkö et al. 2019, 2020).

The development of functional imaging techniques, such as positron emission tomography (PET) and functional magnetic resonance imaging (fMRI), provides a possibility of investigating functional changes of VM. Previous studies have shown that patients with VM often have abnormal vestibular information processing and abnormal functional interactions between visual and vestibular networks in the cerebral cortex during tilt (Wang and Lewis 2016).

At present, task fMRI was primarily used to study brain functions in VM patients. Studies have shown that patients with VM showed increased activation in multiple levels of vestibular integration centers and cortical areas related to higher-level vestibular information processing (Bottini et al. 2001; Russo et al. 2014; Bense et al. 2001; Bucher et al. 1998). Previous task-state fMRI studys in healthy volunteers exposed to magnetic vestibular stimulation (Bottini et al. 2001; Bense et al. 2001)showed that the brain regions involved in balance and spatial navigation are complex networks, including the insula, superior temporal gyrus (STG) and inferior parietal lobule, precentral gyrus and postcentral gyrus, anterior insula,inferior and middle frontal gyrus, anterior cingulate gyrus and hippocampus. Some studies also speculated that the pain and vestibular sense in patients with VM may be related to the thalamus (Bense et al. 2001; Bucher et al. 1998). However, task-related brain regions are sometimes not associated with brain functional changes related to VM itself, which may be associated with brain functional changes related to task stimulation. Therefore, the real-time measurement of spontaneous brain functional activities by using resting-state fMRI can better reflect the pathogenesis of VM (Lee et al. 2013).

Under this background, the present study aimed to investigate functional brain activity alterations among patients with VM, health controls ( $\mathrm{HCs}$ ), and patients with migraine by using regional brain activity measures including amplitude of low frequency fluctuation (ALFF), fractional amplitude of low-frequency fluctuation (fALFF), regional homogeneity (ReHo), and seed-based functional connectivity analysis, and explore its possible mechanisms involving the vestibular pathway. 


\section{Methods \\ Participants}

A total of 17 patients with definite vestibular migraine (dVM) who received treatment in Aerospace Center Hospital between December 2018 and December 2020 were included. Clinical data of all patients were collected. Neurological examination, neuro-orthoptic examination, blood pressure, cardiac function, thyroid function tests, immune-related laboratory tests and lower limb electromyography were performed to exclude other medical diseases (such as transient ischemic attacks, hypertension, autoimmune disease and neuromuscular disease). Videonystagmography, caloric test, head impulse test, and vestibular evoked myogenic potential stimulation were performed to exclude peripheral vestibular lesions. MRI was performed to exclude cerebral lesions and structural abnormalities.

17 age- and gender-matched HCs were included, they had no history of headache or vestibular deficits and had no severe medical diseases. 8 age- and gender-matched patients with migraine were also included in the study, patients with migraine was diagnosed according to the diagnostic criteria of ICHD-III in 2013 (Headache Classification Committee 2013), patients with migraine had no history of vestibular deficits, no severe medical diseases, and they also had no aura.

All subjects underwent peripheral vestibular function evaluation to exclude peripheral vestibular lesions. And handedness examination was performed to exclude left handed participants. Patients with VM (during an interictal phase), HCs, and patients with migraine (during an interictal phase) further scanned for fMRI. This study was approved by the Ethics Committee of Aerospace Center Hospital. All participants volunteered to participate in this study and signed informed consent.

\section{Caloric vestibular test}

During the caloric test, the left and right external auditory canals were irrigated with cold $\left(30^{\circ} \mathrm{C}\right)$ and warm $\left(44^{\circ} \mathrm{C}\right)$ water with the patient in the supine position and their head raised by $30^{\circ}$. The right external auditory canal was first irrigated with warm water, followed by the left external auditory canal with warm water and the right external auditory canal with cold water, and last the left external auditory canal with cold water (i.e., right warm (RW), followed by left warm (LW) and right cold (RC), and last left cold (LC)). There was a 5-min interval between irrigations. The slow-phase velocity (SPV) during irrigation was recorded, and the canal paresis (CP) value was calculated according to the following formula: $C P=(R C+R W-L C-L W) /(R C+R W+L C+L W) \times 100$. A CP value of $>25 \%$ indicates reduced unilateral horizontal semicircular canal function (No authors listed 1999). A sum of the SPV values of the bilateral semicircular canals of $\leq 12 \% / \mathrm{s}$ suggests reduced bilateral horizontal semicircular canal function. The criteria for hyperactive responses were as follows: total peak cool response $(\mathrm{LC}+\mathrm{RC})$ of $>99 \%$, total peak warm response $(\mathrm{LW}+\mathrm{RW})$ of $>146^{\circ} / \mathrm{s}$, total peak response $(\mathrm{LC}+\mathrm{RC}+\mathrm{LW}+\mathrm{RW})$ of $>221^{\circ} / \mathrm{s}$. Caloric test intolerance refers to the main symptoms, including obvious nausea, vomiting, numbness in hands and feet, and body stiffness. Caloric test intolerance was considered severe if its duration was $>1 \mathrm{~h}$.

\section{Image acquisition}

Patients with dVM and migraine were scanned during the interictal phase (at least 7 days after and before an attack). All subjects were scanned using a 3.0-Tesla MR (SIEMENS MAGNETOM Skyra syngo MR D13, Germany) with a 16-channel head and neck coil. During image acquisition, subjects' head was immobilized to avoid head movement. Subjects were asked to relax with their eyes closed, stay awake throughout the scanning. Images were obtained in sagittal plane using a 3D gradient-echo T1WI sequence with the following parameters: 192 sagittal slices; repetition time $=1900 \mathrm{~ms}$; echo time $=2.43 \mathrm{~ms}$; flip angle $=8^{\circ}$; field of view $=256 \mathrm{~mm} \times 256 \mathrm{~mm} \times 256$ $\mathrm{mm}$; Voxel size $=1.0 \mathrm{~mm} \times 1.0 \mathrm{~mm} \times 1.0 \mathrm{~mm}$. Resting-state $\mathrm{fMRI}$ images was obtained using echo-planar imaging (EPI) sequence sequence: repetition time $=2000 \mathrm{~ms}$; echo time $=30 \mathrm{~ms}$; flip angle $=90^{\circ} ;$ field of view $=222 \mathrm{~mm} \times 222 \mathrm{~mm} \times 222 \mathrm{~mm}$; Voxel size: 3.0 $\mathrm{mm} \times 3.0 \mathrm{~mm} \times 3.0 \mathrm{~mm}$. After scanning, a total of 200 volumes were obtained, and the scan time was 6 minutes and 48 seconds. All subjects remained awake during scanning and did not experience significant discomfort during or after scanning.

\section{MRI image data processing}

Image data were preprocessed with the toolbox Data Processing Assistant for Resting-State functional MR imaging (DPARSF; http://www.restfmri.net/forum/DPARSF) on the MATLAB2013 platform (Chao-Gan and Yu-Feng 2010). Brain regions were identified 
using automated anatomical labeling (AAL) atlas. In order to ensure the accuracy of the position information, the subjects who had more than $1.5 \mathrm{~mm}$ head translation and $1.5^{\circ}$ head rotation in $\mathrm{x}-\mathrm{y}$-, or $\mathrm{z}$-direction were excluded.

\section{ALFF analyisis}

ALFF and fALFF refer to the intensity of spontaneous brain activity. The higher the values, the greater the spontaneous brain activity in the brain regions. ALFF analysis was done with following steps: (i) data conversion: image data were converted from DICOM format to NIFTI format that can be processed by SPM12; (ii) removal of the first 10 time points: due to the possible instability of the initial MRI signal caused by $\mathrm{T} 1$ relaxation and the need for participants to adapt to the scanning environment, the first 10 time points were deleted; (iii) time correction: time correction is performed to ensure that all voxels within one volume had been acquired at the same time, the 35th slice was chosen as the reference slice; (iv) head movement correction: slight head motion of the subject between the time points during the scan was corrected, in order to ensure the accuracy of the position information, the subjects who had more than $1.5 \mathrm{~mm}$ head translation in $x-, y-$, or $z$-direction and $1.5^{\circ}$ head rotation were removed; (v) removal of linear drift: linear trend formed due to heating of MR scanner and fatigue in subjects after long MRI scanning time was removed; (vi) regression: a linear regression model was used to remove the interference signal in the BOLD signal; (vii) spatial normalization: to solve the problems related to difference in brain morphology among different subjects and the inconsistencies in spatial position during scanning, $\mathrm{fMRI}$ images were spatially normalized to a standard space using DARTEL and resampled at a resolution of $3 \mathrm{~mm} \times 3 \mathrm{~mm} \times 3 \mathrm{~mm}$; (viii) spatial smoothing: smoothening was conducted with a Gaussian kernel of $8 \mathrm{~mm} \times 8 \mathrm{~mm} \times 8 \mathrm{~mm}$ to reduce registration errors and increase the normality of the data; (ix) ALFF calculation: the power spectrum is obtained by converting signals in time domain into the frequency domain using the Fast Fourier Transform, the average square root of the power spectrum was calculated as the ALFF value of the whole brain; $(x)$ fALFF calculation: fALFF value was calculated at frequency range of $0.01-0.1 \mathrm{~Hz}$.

\section{REHO}

ReHo is defined as the correlation of the time series of a given voxel with those of its nearest neighboring voxels. ReHo analysis was performed with following steps: (i) data format conversion; (ii) removal of first 10 time points; (iii) time correction; (iv) head movement correction; (v) linear drift removal; (vi) regression; (vii) band-pass filtering: the time series for each voxel was filtered (band-pass, 0.01-0.8 $\mathrm{Hz}$ ) to remove low-frequency drift and high frequency noise; (viii) spatial normalization; (ix) REHO calculation: ReHo was calculated by calculating the Kendall's coefficient of concordance (KCC) of the time series of a given voxel with those of its nearest neighbors (Zang et al. 2004); (x) spatial smoothing.

\section{Seed-based FC analysis}

Seed-based FC analysis was performed with following steps: (i) data format conversion; (ii) removal of first 10 frames; (iii) time correction; (iv) head movement correction; (v) linear drift removal; (vi) regression; (vii) band-pass filtering; (viii) spatial normalization; (ix) spatial smoothing; (x) FC calculation: brain regions with altered fALFF were converted into MASKs using Xjview (https://www.alivelearn.net/xjview), and selected as seeds for seeded-based FC analysis to assess FC alterations with other brain regions, particularly especially other vestibular cortex and visual cortex areas.

\section{Statistical analysis}

fMRI data analysis was performed using SPM 12 software. Comparison of the difference in results of ALFF, REHO, FC between groups were tested using a two-sample $T$ test and adjusted for covariates including age and sex. $\mathrm{P}<0.01$ was considered to be statistically different. Multiple comparisons corrections were performed using family-wise-error correction (FWE). Results were visualized using XJVIEW (https://www.alivelearn.net/xjview) and BrainNet (http://www.nitrc.org/projects/bnv/) (Xia et al. 2013).

\section{Results}

\section{Clinical data of patients included in the study}


In the VM group, there are 7 males and 10 females, the average age was $39.47 \pm 9.78$ years (range, 24-58 years). The median length of medical history was 5 years ( $8.12 \pm 7.84$ years, range,1-30 years). As to the frequency of VM attacks, one patient reported one attack per week, 7 patients reported one attack per month, 9 patients reported once attack per year, and all patients had unilateral location of headaches during VM attacks. In the HC group, there were 7 males and 10 females with an average age of $39.82 \pm 13.01$ years, all HCs were right-handed. In the migraine group, there were 3 males and 5 females, all were right-handed, the average age was $41.88 \pm 11.03$ years (Table 2).

All 17 patients with VM had a history of migraine, including 12 (70.6\%) patients of recurrent spontaneous vertigo, 2 (11.7\%) patients of visually-induced vertigo, and $3(17.6 \%)$ patients of head motion-induced vertigo. All 17 patients with VM reported worsening of dizziness during visual stimulation. The accompanying symptoms were photophobia or phonophobia $(n=15,88.2 \%)$, migraine-like headache $(n=8$, $47.1 \%)$, visual aura during VM attacks $(n=7,41.2 \%)$.

During the caloric test, hyperactive response was found in 5 (29.4\%) patients with VM, and caloric test intolerance was found in 12 (70.6\%) patients. Among 17 patients with VM, 11 (64.7\%) patients had a history of motion sickness (Table 1).

\section{ALFF and fALFF results}

Compared with HCs, VM group exhibited significantly increased ALFF and fALFF values in the right temporal lobe, including STG and middle temporal gyrus (MTG) ( $X=51, Y=-54, Z=6, P=0.002$, FWE corrected; $X=69, Y=-45, Z=3 ; P=0.001$, FWE corrected, respectively, Table 3). Increased ALFF values in the right temporal lobe in patients with VM indicated that the intensity of spontaneous functional activity in this region was enhanced.

Compared with migraine group, VM group showed significantly decreased ALFF values in the right median cingulate and paracingulate gyri $(X=21, Y=-30, Z=45, P=0.009$, FWE corrected, Table 3$)$, indicating that the intensity of spontaneous functional activity in this region in patients with VM was weakened. VM group also showed significantly increased fALFF values in the right parietal lobe (postcentral gyrus and superior parietal gyrus) ( $X=24, Y=-45, Z=69, P=0.011$, FWE corrected, Table 3 ), and right frontal lobe (supplementary motor area (SMA) and dorsolateral superior frontal gyrus) $(X=24, Y=-45, Z=69, P=0.026$, FWE corrected, Table 3$)$ compared with migraine group. The results indicated that the intensity of spontaneous functional activity in the above-mentioned brain regions in patients with VM was increased.

Compared with the HC group, migraine group showed significantly increased ALFF values in the right limbic lobe (mainly including the right parahippocampal gyrus and right fusiform gyrus) $(X=33, Y=-18, Z=-33, P=0.04$, FWE corrected), left temporal lobe (left inferior temporal gyrus (ITG)) $(X=57, Y=-36, Z=-27, P=0.024$, FWE corrected) and the right frontal lobe (mainly including SMA, right median cingulate and paracingulate gyri, and right inferior frontal gyrus) $(X=21, Y=-18, Z=54, P \otimes 0.001$, FWE corrected), as well as significantly decreased ALFF values in the pons and brainstem ( $X=6, Y=-33, Z=-33, P=0.013$, FWE corrected, Table 3$)$.

\section{ReHo results}

Patients with VM showed increased ReHo values in the right temporal lobe (STG, MTG and ITG) in comparison with HCs (X=60, Y=-45, $Z=6, P=0.013$, FWE corrected, Table 3). Increased ReHo values in the right temporal lobe indicated an increase in consistency of regional brain activity in this region in patients with VM. Regions with increased ReHo values contain regions with increased ALFF values and adjacent regions. Increased ReHo values and ALFF values were both observed in the right temporal lobe, indicating that the regional functional activities in the right temporal lobe (STG, MTG, and ITG) in patients with VM were enhanced.

Patients with VM showed significantly increased ReHo values in the right thalamus compared with patients with migraine $(X=12, Y=-21$, $Z=-3, P=0.043$, FWE corrected, Table 3), suggesting an increase in consistency of regional brain activity in this region in patients with VM.

Compared with HCs, patients with migraine had significantly decreased ReHo values in the frontal cortex (mainly including left and right SMA, left dorsolateral superior frontal gyrus, left median cingulate and paracingulate gyri, right paracentral lobule, right dorsolateral superior frontal gyrus, left and right middle frontal gyrus) $(X=-18, Y=3, Z=60, P<0.001$, FWE corrected, Table 3).

\section{Results from seed-based FC analysis}


Brain regions with altered fALFF were selected for seeded-based FC analysis. The results revealed significantly increased FC in the right temporal lobe (STG and MTG), small part of the parietal lobe (inferior parietal lobe) and the insular lobe in patients with VM compared with the HC group ( $X=51, Y=-45, Z=15, P=0.016$, FWE corrected). No significant difference was found between migraine and HC groups, as well as VM and migraine groups.

\section{Discussion}

VM is one of the common causes of episodic central vestibular disorders, which affects more than $1 \%$ of the general population (Stolte et al. 2015). Generally, VM is a clinical diagnosis lacking positive strong affirmative diagnostic patterns in each patient. The underlying mechanisms of VM are still unclear. Whether vestibular migraine belongs to the migraine subtype or is a separate disorder deserves further study. Clinically, the vestibular symptoms are very prominent in patients with VM. In this study, we found that majority of patients with VM (12/17) had spontaneous vertigo, which was mostly triggered by head or visual movements. The result is similar to the findings of Neuhauser et al (2006) and Cho et al (2016). In fact, the heterogeneity of vestibular symptoms may be related to patients' subjective description. In this study, it is interesting that most patients with VM (70.6\%) developed caloric test intolerance, this may be due to increased sensitivity of the vestibule (Boldingh e al. 2011; Heuberger et al. 2014). Caloric test intolerance has been repeatedly reported in previous studies, patients usually developed severe intolerance during irrigation with warm or cold water, such as obvious nausea, vomiting, heart palpitations, numbness of hands and feet, and even body stiffness. In some patients, those symptoms may even take several hours to recover (Polensek and Tusa 2010; von Brevern et al. 2005; Teggi et al. 2009; Jeong et al. 2010; Kayan and Hood 1984). In addition, we found that $64.7 \%$ patients with VM had motion sickness. The correlation between motion sickness and VM has been reported by Akdal et al. (2015), their findings showed that motion sickness was more common in migraine accompanied by vertigo compared with patients with migraine (75\% vs 50\%). Langhagen et al. also found that $51 \%$ patients with VM had a history of motion sickness (Abouzari et al. 2020). Therefore, obtaining history of motion sickness may contribute to clinical diagnosis of VM (Lipson et al. 2020; Langhagen et al. 2015).

In this study, migraine without aura was most common in patients with VM. The most common migraine-like symptoms in VM patients was photophobia/phonophobia $(n=15,88.2 \%)$, followed by migraine-like headache $(n=8,47.1 \%)$, and visual aura ( $n=7,41.2 \%)$. This result is consistent with previous studies made by Zhang et al. (2016) and Cho et al. (2016).

In this study, we found that compared with HCs, spontaneous functional activity in the right temporal lobe (STG and MTG), ReHo values in the right temporal lobe (STG, MTG and ITG), and FC within the right temporal lobe were both enhanced in patients with VM, suggesting enhanced regional functional activity in the right temporal lobe in patients with VM. This may be related to the hearing and visual hypersensitivity (Gollion 2021).

A study has shown that lesions in regions within the temporal lobe such as STG (AAL; BA22), MTG, and ITG are often associated with allocentric deficits (Grimsen et al. 2008). Allocentric processing is known as allocentric spatial discrimination ability, i.e., impaired stimulus-centered advanced navigation system. Medina et al. (2009) performed MRI and spatial cognition tests on 171 patients with right supratentorial ischemic stroke and found that parts of the dorsal stream of visual processing, including the right supramarginal gyrus, are involved in spatial encoding in egocentric coordinates, parts of the ventral stream, including the posterior ITG, are involved in allocentric processing. Shah-Basak et al. (2018) performed repetitive transcranial magnetic stimulation (rTMS) on the right STG $(48,-20,-8)$ in 11 right-handed healthy subjects, revealed impaired stimulus-centered spatial cognition in patients. Patients with VM in the present study are similar to healthy subjects who underwent rTMS on right STG in the above-mentioned study of Shah-Basak et al, indicating that ventral stream of visual processing and allocentric spatial cognition were impaired in patients with VM. We speculated that enhanced spontaneous functional activity in the right temporal lobe (STG, MTG and ITG) in patients with VM may be related to patients' discomfort and worsening of dizziness during visual stimulation. Enhanced regional functional activity of MTG in patients with VM is presumed to be related with auditory information processing (Xu et al. 2019).

Furthermore, we found that patients with VM showed increased regional spontaneous activity in the right parietal lobe-frontal lobethalamus. (1) Compared with patients with migraine, spontaneous functional activity in the right parietal lobe (postcentral gyrus and superior parietal gyrus) $(X=24, Y=-45, Z=69)$ and right frontal lobe (SMA and dorsolateral superior frontal gyrus) ( $X=24, Y=-45, Z=69)$ was enhanced in patients with VM. It is speculated that patients with VM have abnormalities in visual-spatial information integration, and higher-level vestibular cortical integration areas are in a sensitive state. Previous studies based on working memory have shown that the dorsolateral superior frontal gyrus and superior parietal lobule (superior parietal gyrus) received visual and spatial sensory stimuli and integrate visual information flow and spatial information (vestibular information related to position), the right frontal lobe is related to visual-spatial tasks (Koenigs et al. 2011). Generally, visual-spatial information integration requires the combined effects of top-down

Page 6/17 
(cognitive control and goal-directed) modulation and bottom-up inputs (capture of external sensory stimuli) in the superior parietal lobule (Pinto et al. 2013; Wu et al. 2018). Postle et al. (2006) applied rTMS stimulation over superior parietal gyrus, and found that subjects' performance was not stable during tasks requiring visual-spatial coordination, and changes in the parietofrontal network is observed, application of rTMS on superior parietal gyrus is similar to enhanced spontaneous activity observed in patients with VM in this study. (2) Compared with patients with migraine, increase in consistency of regional brain activity in the right thalamus was observed in patients with VM. The thalamus is the relay station for all sensory input, it is also considered as an integrated area of multisensory vestibular information (Brandt and Dieterich 2019). As mentioned above, compared with patients with migraine, VM patients showed enhanced regional functional activity in the thalamus, right parietal lobe (postcentral gyrus and superior parietal gyrus) and right frontal lobe (SMA and dorsolateral superior frontal gyrus). It is speculated that the vestibular pathway, right parietal lobe-frontal lobe-thalamus, in patients with VM is in a sensitive state, this may be related to the repeated episodes of vertigo and worsening of dizziness during visual stimulation that are different from those of patients with migraine. Versino et al. also found that VM mainly impairs the capability to integrate different vestibular stimuli (Versino et al. 2020).

In this study, we also compared the differences in the regional brain activity measures between patients with migraine and HCs. The results showed that enhanced spontaneous functional activity in the right limbic lobe (mainly including the right parahippocampal gyrus and the right fusiform gyrus) and right frontal lobe (SMA, right median cingulate and paracingulate gyri, and right inferior frontal gyrus), decreased spontaneous function activity in the pons and brainstem, as well as reduced ReHo values in the frontal cortex in patients with migraine compared with HCs. This results is consistent with the results of previous fMRI study showing that brain stem and hippocampus play a key role in the onset of migraine attack, and the limbic lobe is involved in the formation of migraine-related pain network, abnormal frontal lobe function can eventually lead to reduced pain threshold in patients with migraine (Russo et al. 2017). Our findings showed that brain regions with altered functional activity in patients with VM are very different from those in patients with migraine relative to $\mathrm{HC}$ groups, there appears to be little overlap, and the mechanisms involved may be different.

\section{Limitations}

This study has some limitations: the sample size of the study is small, which limits the generalization of the results; we did not assess visual vertigo and multisensory vestibular integration in patients with VM included in this study, further behavioral and vestibular/visual stimulation researches are needed to verify our findings.

\section{Conclusions}

Patients with VM showed enhanced spontaneous functional activity in the right temporal lobe (STG, MTG and ITG) compared with HCs, they also showed increased spontaneous activity in the right parietal lobe-frontal lobe-thalamus compared with patients with migraine. Patients with VM and migraine both had altered brain function, but the regions involved are different. Our findings provide evidence for the regional functional properties of brain regions in patients with VM and for further functional connectivity studies based on ROI of multilevel vestibular pathways, as well as brain network connectome studies based on small-world brain graph theory.

\section{Declarations}

\section{-Ethical Approval}

This study was approved by the Ethics Committee of Aerospace Center Hospital, Peking University Aerospace School of Clinical Medicine.

\section{-Consent to Participate}

The patients/participants provided their written informed consent to participate in this study.

\section{-Consent to Publish}

Not applicable. 


\section{-Authors Contributions}

XY designed the study. ZL, LS, BS, XL performed the experiments and analyzed the data. ZL prepared the manuscript. All authors read and approved the final manuscript.

\section{-Funding}

The study was supported by Aerospace Center Hospital (Grant No. YN201802).

\section{-Competing Interests}

The authors declare no competing interests.

\section{-Availability of data and materials}

The datasets used and analyzed during the current study available from the corresponding author on reasonable request.

\section{References}

1. Abouzari, M., Cheung, D., Pham, T., Goshtasbi, K., Sarna, B., Tajran, S., Sahyouni, S., Lin, H. W., \& Djalilian, H. R. (2020). The Relationship Between Vestibular Migraine and Motion Sickness Susceptibility. Otology \& neurotology : official publication of the American Otological Society, American Neurotology Society [and] European Academy of Otology and Neurotology, 41(8), 11161121. https://sci-hub.se/10.1097/MA0.0000000000002705

2. Agarwal, K., Bronstein, A. M., Faldon, M. E., Mandalà, M., Murray, K., \& Silove, Y. (2012). Visual dependence and BPPV. Journal of neurology, 259(6), 1117-1124. https://doi.org/10.1007/s00415-011-6311-7

3. Akdal, G., Baykan, B., Erta?, M., Zarifo?lu, M., Karli, N., Saip, S., \& Siva, A. (2015). Population-based study of vestibular symptoms in migraineurs. Acta oto-laryngologica, 135(5), 435-439. https://doi.org/10.3109/00016489.2014.969382

4. Balaban C. D. (2011). Migraine, vertigo and migrainous vertigo: Links between vestibular and pain mechanisms. Journal of vestibular research : equilibrium \& orientation, 21(6), 315-321. https://doi.org/10.3233/VES-2011-0428

5. Bense, S., Stephan, T., Yousry, T. A., Brandt, T., \& Dieterich, M. (2001). Multisensory cortical signal increases and decreases during vestibular galvanic stimulation (fMRI). Journal of neurophysiology, 85(2), 886-899. https://doi.org/10.1152/jn.2001.85.2.886

6. Boldingh, M. I., Lj?stad, U., Mygland, A., \& Monstad, P. (2011). Vestibular sensitivity in vestibular migraine: VEMPs and motion sickness susceptibility. Cephalalgia : an international journal of headache, 31(11), 1211-1219.

https://doi.org/10.1177/0333102411409074

7. Bottini, G., Karnath, H. O., Vallar, G., Sterzi, R., Frith, C. D., Frackowiak, R. S., \& Paulesu, E. (2001). Cerebral representations for egocentric space: Functional-anatomical evidence from caloric vestibular stimulation and neck vibration. Brain : a journal of neurology, 124(Pt 6), 1182-1196. https://doi.org/10.1093/brain/124.6.1182

8. Brandt, T., \& Dieterich, M. (2019). Thalamocortical network: a core structure for integrative multimodal vestibular functions. Current opinion in neurology, 32(1), 154-164. https://doi.org/10.1097/WC0.0000000000000638

9. Bucher, S. F., Dieterich, M., Wiesmann, M., Weiss, A., Zink, R., Yousry, T. A., \& Brandt, T. (1998). Cerebral functional magnetic resonance imaging of vestibular, auditory, and nociceptive areas during galvanic stimulation. Annals of neurology, 44(1), 120-125. https://doi.org/10.1002/ana.410440118

10. Chao-Gan, Y., \& Yu-Feng, Z. (2010). DPARSF: A MATLAB Toolbox for "Pipeline" Data Analysis of Resting-State fMRI. Frontiers in systems neuroscience, 4, 13. https://sci-hub.se/10.3389/fnsys.2010.00013

11. Cho, S. J., Kim, B. K., Kim, B. S., Kim, J. M., Kim, S. K., Moon, H. S., Song, T. J., Cha, M. J., Park, K. Y., \& Sohn, J. H. (2016). Vestibular migraine in multicenter neurology clinics according to the appendix criteria in the third beta edition of the International Classification of Headache Disorders. Cephalalgia : an international journal of headache, 36(5), 454-462.

https://doi.org/10.1177/0333102415597890 
12. Dieterich, M., Obermann, M., \& Celebisoy, N. (2016). Vestibular migraine: the most frequent entity of episodic vertigo. Journal of neurology, 263 Suppl 1, S82-S89. https://doi.org/10.1007/s00415-015-7905-2

13. Furman, J. M., Marcus, D. A., \& Balaban, C. D. (2013). Vestibular migraine: clinical aspects and pathophysiology. The Lancet. Neurology, 12(7), 706-715. https://doi.org/10.1016/S1474-4422(13)70107-8

14. Grimsen, C., Hildebrandt, H., \& Fahle, M. (2008). Dissociation of egocentric and allocentric coding of space in visual search after right middle cerebral artery stroke. Neuropsychologia, 46(3), 902-914. https://doi.org/10.1016/j.neuropsychologia.2007.11.028

15. Headache Classification Committee of the International Headache Society (IHS) (2013). The International Classification of Headache Disorders, 3rd edition (beta version). Cephalalgia : an international journal of headache, 33(9), 629-808. https://doi.org/10.1177/0333102413485658

16. Headache Classification Committee of the International Headache Society (IHS) (2013). The International Classification of Headache Disorders, 3rd edition (beta version). Cephalalgia : an international journal of headache, 33(9), 629-808. https://doi.org/10.1177/0333102413485658

17. Heuberger, M., Sa?lam, M., Todd, N. S., Jahn, K., Schneider, E., \& Lehnen, N. (2014). Covert anti-compensatory quick eye movements during head impulses. PloS one, 9(4), e93086. https://doi.org/10.1371/journal.pone.0093086

18. Jeong, S. H., Oh, S. Y., Kim, H. J., Koo, J. W., \& Kim, J. S. (2010). Vestibular dysfunction in migraine: effects of associated vertigo and motion sickness. Journal of neurology, 257(6), 905-912. https://doi.org/10.1007/s00415-009-5435-5

19. Kayan, A., \& Hood, J. D. (1984). Neuro-otological manifestations of migraine. Brain : a journal of neurology, 107 ( Pt 4 ), $1123-1142$. https://doi.org/10.1093/brain/107.4.1123

20. Koenigs, M., Acheson, D. J., Barbey, A. K., Solomon, J., Postle, B. R., \& Grafman, J. (2011). Areas of left perisylvian cortex mediate auditory-verbal short-term memory. Neuropsychologia, 49(13), 3612-3619. https://doi.org/10.1016/j.neuropsychologia.2011.09.013

21. Langhagen, T., Lehrer, N., Borggraefe, I., Heinen, F., \& Jahn, K. (2015). Vestibular migraine in children and adolescents: clinical findings and laboratory tests. Frontiers in neurology, 5, 292. https://doi.org/10.3389/fneur.2014.00292

22. Lee, M. H., Smyser, C. D., \& Shimony, J. S. (2013). Resting-state fMRI: a review of methods and clinical applications. AJNR. American journal of neuroradiology, 34(10), 1866-1872. https://doi.org/10.3174/ajnr.A3263

23. Lempert, T., Olesen, J., Furman, J., Waterston, J., Seemungal, B., Carey, J., Bisdorff, A., Versino, M., Evers, S., \& Newman-Toker, D. (2012). Vestibular migraine: diagnostic criteria. Journal of vestibular research : equilibrium \& orientation, 22(4), 167-172. https://doi.org/10.3233/VES-2012-0453

24. Lipson, S., Wang, A., Corcoran, M., Zhou, G., \& Brodsky, J. R. (2020). Severe motion sickness in infants and children. European journal of paediatric neurology : EJPN : official journal of the European Paediatric Neurology Society, 28, 176-179. https://scihub.se/10.1016/j.ejpn.2020.06.010

25. Medina, J., Kannan, V., Pawlak, M. A., Kleinman, J. T., Newhart, M., Davis, C., Heidler-Gary, J. E., Herskovits, E. H., \& Hillis, A. E. (2009). Neural substrates of visuospatial processing in distinct reference frames: evidence from unilateral spatial neglect. Journal of cognitive neuroscience, 21(11), 2073-2084. https://doi.org/10.1162/jocn.2008.21160

26. Neuhauser, H. K., Radtke, A., von Brevern, M., Feldmann, M., Lezius, F., Ziese, T., \& Lempert, T. (2006). Migrainous vertigo: prevalence and impact on quality of life. Neurology, 67(6), 1028-1033. https://doi.org/10.1212/01.wnl.0000237539.09942.06

27. No authors listed. Caloric test protocol. (1999). British journal of audiology, 33(3), 179-184.

28. Pinto, Y., van der Leij, A. R., Sligte, I. G., Lamme, V. A., \& Scholte, H. S. (2013). Bottom-up and top-down attention are independent. Journal of vision, 13(3), 16. https://doi.org/10.1167/13.3.16

29. Polensek, S. H., \& Tusa, R. J. (2010). Nystagmus during attacks of vestibular migraine: an aid in diagnosis. Audiology \& neurootology, 15(4), 241-246. https://doi.org/10.1159/000255440

30. Postle, B. R., Ferrarelli, F., Hamidi, M., Feredoes, E., Massimini, M., Peterson, M., Alexander, A., \& Tononi, G. (2006). Repetitive transcranial magnetic stimulation dissociates working memory manipulation from retention functions in the prefrontal, but not posterior parietal, cortex. Journal of cognitive neuroscience, 18(10), 1712-1722. https://doi.org/10.1162/jocn.2006.18.10.1712

31. Pyykkö, I., Manchaiah, V., Färkkilä, M., Kentala, E., \& Zou, J. (2019). Association between Ménière's disease and vestibular migraine. Auris, nasus, larynx, 46(5), 724-733. https://sci-hub.se/10.1016/j.anl.2019.02.002

32. Pyykkö, I., Pyykkö, N., \& Manchaiah, V. (2020). Vestibular drop attacks in Ménière's disease and its association with migraine. European archives of oto-rhino-laryngology : official journal of the European Federation of Oto-Rhino-Laryngological Societies (EUFOS) : affiliated with the German Society for Oto-Rhino-Laryngology - Head and Neck Surgery, 277(7), 1907-1916. https://scihub.se/10.1007/s00405-020-05890-3 
33. Russo, A., Marcelli, V., Esposito, F., Corvino, V., Marcuccio, L., Giannone, A., Conforti, R., Marciano, E., Tedeschi, G., \& Tessitore, A. (2014). Abnormal thalamic function in patients with vestibular migraine. Neurology, 82(23), 2120-2126. https://doi.org/10.1212/WNL.0000000000000496

34. Russo, A., Silvestro, M., Tedeschi, G., \& Tessitore, A. (2017). Physiopathology of Migraine: What Have We Learned from Functional Imaging?. Current neurology and neuroscience reports, 17(12), 95. https://doi.org/10.1007/s11910-017-0803-5

35. Shah-Basak, P. P., Chen, P., Caulfield, K., Medina, J., \& Hamilton, R. H. (2018). The role of the right superior temporal gyrus in stimuluscentered spatial processing. Neuropsychologia, 113,6-13. https://doi.org/10.1016/j.neuropsychologia.2018.03.027

36. Stolte, B., Holle, D., Naegel, S., Diener, H. C., \& Obermann, M. (2015). Vestibular migraine. Cephalalgia : an international journal of headache, 35(3), 262-270. https://doi.org/10.1177/0333102414535113

37. Teggi, R., Colombo, B., Bernasconi, L., Bellini, C., Comi, G., \& Bussi, M. (2009). Migrainous vertigo: results of caloric testing and stabilometric findings. Headache, 49(3), 435-444. https://doi.org/10.1111/j.1526-4610.2009.01338.x

38. Versino, M., Mandalà, M., Colnaghi, S., Ricci, G., Faralli, M., \& Ramat, S. (2020). The integration of multisensory motion stimuli is impaired in vestibular migraine patients. Journal of neurology, 267(10), 2842-2850. https://sci-hub.se/10.1007/s00415-020-099051

39. von Brevern, M., Zeise, D., Neuhauser, H., Clarke, A. H., \& Lempert, T. (2005). Acute migrainous vertigo: clinical and oculographic findings. Brain : a journal of neurology, 128(Pt 2), 365-374. https://doi.org/10.1093/brain/awh351

40. Wang, J., \& Lewis, R. F. (2016). Abnormal Tilt Perception During Centrifugation in Patients with Vestibular Migraine. Journal of the Association for Research in Otolaryngology : JARO, 17(3), 253-258. https://doi.org/10.1007/s10162-016-0559-7

41. Wu, Z., Mazzola, C. A., Catania, L., Owoeye, O., Yaramothu, C., Alvarez, T., Gao, Y., \& Li, X. (2018). Altered cortical activation and connectivity patterns for visual attention processing in young adults post-traumatic brain injury: A functional near infrared spectroscopy study. CNS neuroscience \& therapeutics, 24(6), 539-548. https://doi.org/10.1111/cns.12811

42. Xia, M., Wang, J., \& He, Y. (2013). BrainNet Viewer: a network visualization tool for human brain connectomics. PloS one, 8(7), e68910. https://sci-hub.se/10.1371/journal.pone.0068910

43. Xu, J., Lyu, H., Li, T., Xu, Z., Fu, X., Jia, F., Wang, J., \& Hu, Q. (2019). Delineating functional segregations of the human middle temporal gyrus with resting-state functional connectivity and coactivation patterns. Human brain mapping, 40(18), 5159-5171. https://doi.org/10.1002/hbm.24763

44. Zang, Y., Jiang, T., Lu, Y., He, Y., \& Tian, L. (2004). Regional homogeneity approach to fMRI data analysis. Neurolmage, 22(1), 394400. https://sci-hub.se/10.1016/j.neuroimage.2003.12.030

45. Zhang, Y., Kong, Q., Chen, J., Li, L., Wang, D., \& Zhou, J. (2016). International Classification of Headache Disorders 3rd edition betabased field testing of vestibular migraine in China: Demographic, clinical characteristics, audiometric findings and diagnosis statues. Cephalalgia : an international journal of headache, 36(3), 240-248. https://doi.org/10.1177/0333102415587704

\section{Tables}

Table 1

Clinical characteristics of patients with patients with vestibular migraine 


\begin{tabular}{|c|c|c|c|c|c|c|c|}
\hline \multirow[t]{2}{*}{ ID/sex/age } & \multirow{2}{*}{$\begin{array}{l}\text { Recurrent } \\
\text { spontaneous } \\
\text { vertigo }\end{array}$} & \multicolumn{3}{|c|}{ Accompanying migraine features } & \multicolumn{2}{|l|}{ Caloric test } & \multirow{2}{*}{$\begin{array}{l}\text { Motion } \\
\text { sickness }\end{array}$} \\
\hline & & $\begin{array}{l}\text { Photophobia } \\
\text { /Phonophobia }\end{array}$ & $\begin{array}{l}\text { Migraine-like } \\
\text { headache }\end{array}$ & $\begin{array}{l}\text { Visual } \\
\text { aura }\end{array}$ & $\begin{array}{l}\text { Hyperactive } \\
\text { response }\end{array}$ & $\begin{array}{l}\text { Caloric test } \\
\text { intolerance }\end{array}$ & \\
\hline \multicolumn{2}{|l|}{ 1/F/52 } & \multicolumn{5}{|l|}{$\sqrt{ }$} & \\
\hline $2 / M / 24$ & $\sqrt{ }$ & $\sqrt{ }$ & $\sqrt{ }$ & $\sqrt{ }$ & & $\sqrt{ }$ & $\sqrt{ }$ \\
\hline 3/F/30 & $\sqrt{ }$ & $\sqrt{ }$ & & & & & $\sqrt{ }$ \\
\hline 4/M/47 & $\sqrt{ }$ & $\sqrt{ }$ & & & & $\sqrt{ }$ & $\sqrt{ }$ \\
\hline $5 / \mathrm{M} / 40$ & $\sqrt{ }$ & $\sqrt{ }$ & & & & $\sqrt{ }$ & $\sqrt{ }$ \\
\hline \multicolumn{2}{|l|}{ 6/M/25 } & $\sqrt{ }$ & $\sqrt{ }$ & & $\sqrt{ }$ & $\sqrt{ }$ & $\sqrt{ }$ \\
\hline 7/F/38 & $\sqrt{ }$ & $\sqrt{ }$ & $\sqrt{ }$ & $\sqrt{ }$ & & $\sqrt{ }$ & $\sqrt{ }$ \\
\hline \multicolumn{2}{|l|}{ 8/M/39 } & \multicolumn{3}{|l|}{$\sqrt{ }$} & $\sqrt{ }$ & \multicolumn{2}{|l|}{$\sqrt{ }$} \\
\hline 9/F/35 & $\sqrt{ }$ & \multicolumn{2}{|l|}{$\sqrt{ }$} & & & \multicolumn{2}{|l|}{$\sqrt{ }$} \\
\hline \multicolumn{2}{|l|}{ 10/F/34 } & \multirow[t]{2}{*}{$\sqrt{ }$} & $\sqrt{ }$ & $\sqrt{ }$ & $\sqrt{ }$ & \multirow[t]{2}{*}{$\sqrt{ }$} & $\sqrt{ }$ \\
\hline $11 / F / 58$ & $\sqrt{ }$ & & $\sqrt{ }$ & & & & $\sqrt{ }$ \\
\hline 12/M/35 & $\sqrt{ }$ & \multicolumn{3}{|l|}{$\sqrt{ }$} & & \multicolumn{2}{|l|}{$\sqrt{ }$} \\
\hline $13 / F / 47$ & $\sqrt{ }$ & $\sqrt{ }$ & $\sqrt{ }$ & $\sqrt{ }$ & $\sqrt{ }$ & $\sqrt{ }$ & $\sqrt{ }$ \\
\hline 14/F/54 & $\sqrt{ }$ & $\sqrt{ }$ & $\sqrt{ }$ & $\sqrt{ }$ & $\sqrt{ }$ & $\sqrt{ }$ & $\sqrt{ }$ \\
\hline \multicolumn{2}{|l|}{ 15/F/44 } & \multicolumn{6}{|l|}{$\sqrt{ }$} \\
\hline 16/F/38 & $\sqrt{ }$ & $\sqrt{ }$ & & $\sqrt{ }$ & & & $\sqrt{ }$ \\
\hline 17/M/31 & $\sqrt{ }$ & & $\sqrt{ }$ & $\sqrt{ }$ & & $\sqrt{ }$ & \\
\hline \multicolumn{8}{|c|}{ 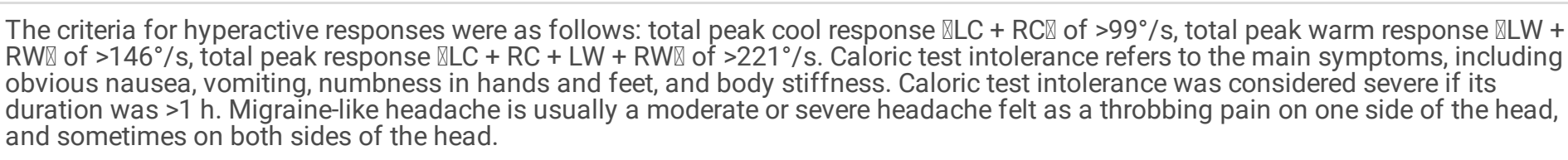 } \\
\hline
\end{tabular}

Table 2

Clinical characteristics of patients with migraine without aura

\begin{tabular}{|c|c|c|c|c|c|c|c|c|}
\hline \multirow{3}{*}{$\begin{array}{l}\text { ID/sex/age } \\
\text { 1/F/52 }\end{array}$} & \multirow{3}{*}{ 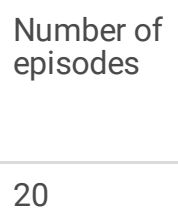 } & \multicolumn{4}{|c|}{ Headache characteristics } & \multicolumn{3}{|c|}{ Accompanying symptoms } \\
\hline & & \multirow{2}{*}{$\begin{array}{l}\text { Unilateral } \\
\text { location } \\
\sqrt{ }\end{array}$} & \multirow[t]{2}{*}{$\begin{array}{l}\text { Pulsating } \\
\text { quality }\end{array}$} & \multirow{2}{*}{$\begin{array}{l}\text { Pain } \\
\text { intensity } \\
\text { severe }\end{array}$} & \multirow{2}{*}{$\begin{array}{l}\text { Causing avoidance of } \\
\text { routine physical activity } \\
\sqrt{ }\end{array}$} & \multicolumn{2}{|c|}{$\begin{array}{l}\text { Nausea/ } \\
\text { vomit }\end{array}$} & \multirow[t]{2}{*}{$\begin{array}{l}\text { Photophobia/ } \\
\text { phonophobia }\end{array}$} \\
\hline & & & & & & $\sqrt{ }$ & $\sqrt{ }$ & \\
\hline 2/M/40 & 14 & $\sqrt{ }$ & $\sqrt{ }$ & moderate & & & $\sqrt{ }$ & \\
\hline $3 / \mathrm{M} / 27$ & 8 & $\sqrt{ }$ & $\sqrt{ }$ & severe & $\sqrt{ }$ & $\sqrt{ }$ & $\sqrt{ }$ & \\
\hline 4/F/44 & 30 & $\sqrt{ }$ & $\sqrt{ }$ & moderate & & $\sqrt{ }$ & $\sqrt{ }$ & \\
\hline 5/F/48 & 13 & $\sqrt{ }$ & $\sqrt{ }$ & moderate & & & $\sqrt{ }$ & \\
\hline 6/F/26 & 34 & & $\sqrt{ }$ & severe & $\sqrt{ }$ & $\sqrt{ }$ & $\sqrt{ }$ & \\
\hline 7/F/41 & 26 & $\sqrt{ }$ & $\sqrt{ }$ & moderate & & & $\sqrt{ }$ & \\
\hline 8/M/57 & 45 & $\sqrt{ }$ & $\sqrt{ }$ & moderate & $\sqrt{ }$ & & $\sqrt{ }$ & \\
\hline
\end{tabular}


Table 3

Altered regional brain functional activity in patients with VM measured by fMRI 


\begin{tabular}{|c|c|c|c|c|c|c|}
\hline \multicolumn{2}{|c|}{$\begin{array}{l}\text { Comparisons between } \\
\text { groups }\end{array}$} & \multicolumn{2}{|l|}{ Regions } & \multirow{3}{*}{$\begin{array}{l}\text { Cluster } \\
\text { size (voxels) }\end{array}$} & \multirow{3}{*}{$\begin{array}{l}\text { T } \\
\text { scores } \\
\text { of } \\
\text { peak } \\
\text { voxel } \\
5.69\end{array}$} & \multirow{3}{*}{$\begin{array}{l}\text { Coordinates } \\
\text { of peak } \\
\text { voxel in } \\
\text { MNI space } \\
(x, y, z) \\
51,-54,6\end{array}$} \\
\hline \multirow{7}{*}{$\begin{array}{l}\text { Patients with } \\
\text { vestibular } \\
\text { migraine vs. health } \\
\text { controls }\end{array}$} & \multirow[t]{2}{*}{ ALFF } & \multirow[t]{2}{*}{ Right temporal lobe } & $\begin{array}{l}\text { Superior temporal } \\
\text { gyrus }\end{array}$ & & & \\
\hline & & & $\begin{array}{l}\text { Middle temporal } \\
\text { gyrus }\end{array}$ & & & \\
\hline & \multirow[t]{2}{*}{ fALFF } & \multirow[t]{2}{*}{ Right temporal lobe } & $\begin{array}{l}\text { Superior temporal } \\
\text { gyrus }\end{array}$ & \multirow[t]{2}{*}{77} & \multirow[t]{2}{*}{4.91} & \multirow[t]{2}{*}{$69,-45,3$} \\
\hline & & & $\begin{array}{l}\text { Middle temporal } \\
\text { gyrus }\end{array}$ & & & \\
\hline & \multirow[t]{3}{*}{$\mathrm{ReHo}$} & \multirow[t]{3}{*}{ Right temporal lobe } & $\begin{array}{l}\text { Superior temporal } \\
\text { gyrus }\end{array}$ & \multirow[t]{3}{*}{136} & \multirow[t]{3}{*}{4.88} & \multirow[t]{3}{*}{$60,-45,6$} \\
\hline & & & $\begin{array}{l}\text { Middle temporal } \\
\text { gyrus }\end{array}$ & & & \\
\hline & & & $\begin{array}{l}\text { Inferior temporal } \\
\text { gyrus }\end{array}$ & & & \\
\hline \multirow{6}{*}{$\begin{array}{l}\text { Patients with } \\
\text { vestibular } \\
\text { migraine } \\
\text { vs. patients with } \\
\text { migraine }\end{array}$} & ALFF & \multicolumn{5}{|l|}{ Gray matter cluster was too small } \\
\hline & \multirow[t]{4}{*}{ fALFF } & \multirow[t]{2}{*}{ Right frontal lobe } & $\begin{array}{l}\text { Right supplementary } \\
\text { motor area }\end{array}$ & \multirow[t]{2}{*}{36} & \multirow[t]{2}{*}{4.86} & \multirow[t]{2}{*}{$12,-9,63$} \\
\hline & & & $\begin{array}{l}\text { Right superior } \\
\text { frontal gyrus, } \\
\text { dorsolateral }\end{array}$ & & & \\
\hline & & \multirow[t]{2}{*}{ Right parietal lobe } & $\begin{array}{l}\text { Right postcentral } \\
\text { gyrus }\end{array}$ & \multirow[t]{2}{*}{43} & \multirow[t]{2}{*}{4.62} & \multirow[t]{2}{*}{$24,-45,69$} \\
\hline & & & $\begin{array}{l}\text { Right superior } \\
\text { parietal gyrus }\end{array}$ & & & \\
\hline & ReHo & Midbrain/tight brainstem/thalamus & Right thalamus & 92 & 4.76 & $12,-21,-3$ \\
\hline \multirow[t]{10}{*}{$\begin{array}{l}\text { Patients with } \\
\text { migraine vs. health } \\
\text { controls }\end{array}$} & \multirow[t]{7}{*}{ ALFF } & \multirow[t]{2}{*}{ Right limbic lobe } & $\begin{array}{l}\text { Right } \\
\text { parahippocampal } \\
\text { gyrus }\end{array}$ & \multirow[t]{2}{*}{45} & \multirow[t]{2}{*}{6.24} & $33,-18,-33$ \\
\hline & & & Right fusiform gyrus & & & \\
\hline & & Left temporal lobe & $\begin{array}{l}\text { Left Inferior } \\
\text { temporal gyrus }\end{array}$ & 51 & 5.53 & $-57,-36,-27$ \\
\hline & & Frontal lobe & $\begin{array}{l}\text { Right supplementary } \\
\text { motor area }\end{array}$ & 125 & 5.09 & $21-1854$ \\
\hline & & & $\begin{array}{l}\text { Median cingulate } \\
\text { and paracingulate } \\
\text { gyri }\end{array}$ & & & \\
\hline & & & $\begin{array}{l}\text { Right superior } \\
\text { frontal gyrus, } \\
\text { dorsolateral }\end{array}$ & & & \\
\hline & & Pons\& brainstem & Pons\& brainstem & 58 & -5.56 & $6,-33,-33$ \\
\hline & ReHo & Frontal lobe & $\begin{array}{l}\text { Right supplementary } \\
\text { motor area }\end{array}$ & 737 & -9.62 & $-18,3,60$ \\
\hline & & & $\begin{array}{l}\text { Left supplementary } \\
\text { motor area }\end{array}$ & & & \\
\hline & & & $\begin{array}{l}\text { Left median } \\
\text { cingulate and } \\
\text { paracingulate gyri }\end{array}$ & & & \\
\hline
\end{tabular}


Right paracentral

lobule

Right superior

frontal gyrus,

dorsolateral

Right middle fronta

gyrus

Left middle frontal

gyrus

ALFF, amplitude of low frequency fluctuation; fALFF, fractional amplitude of low frequency fluctuation; ReHo, regional homogeneity

\section{Figures}
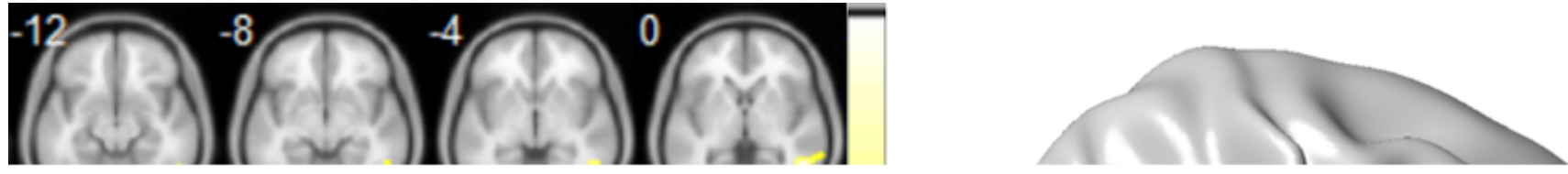

\section{Figure 1}

Differences in ALFF values between three groups. Vestibular migraine (VM) group exhibited significantly increased ALFF values in the right temporal lobe compared with health control $(\mathrm{HC})$ group $(X=51, Y=-54, Z=6)$, indicating enhanced spontaneous functional activity in 

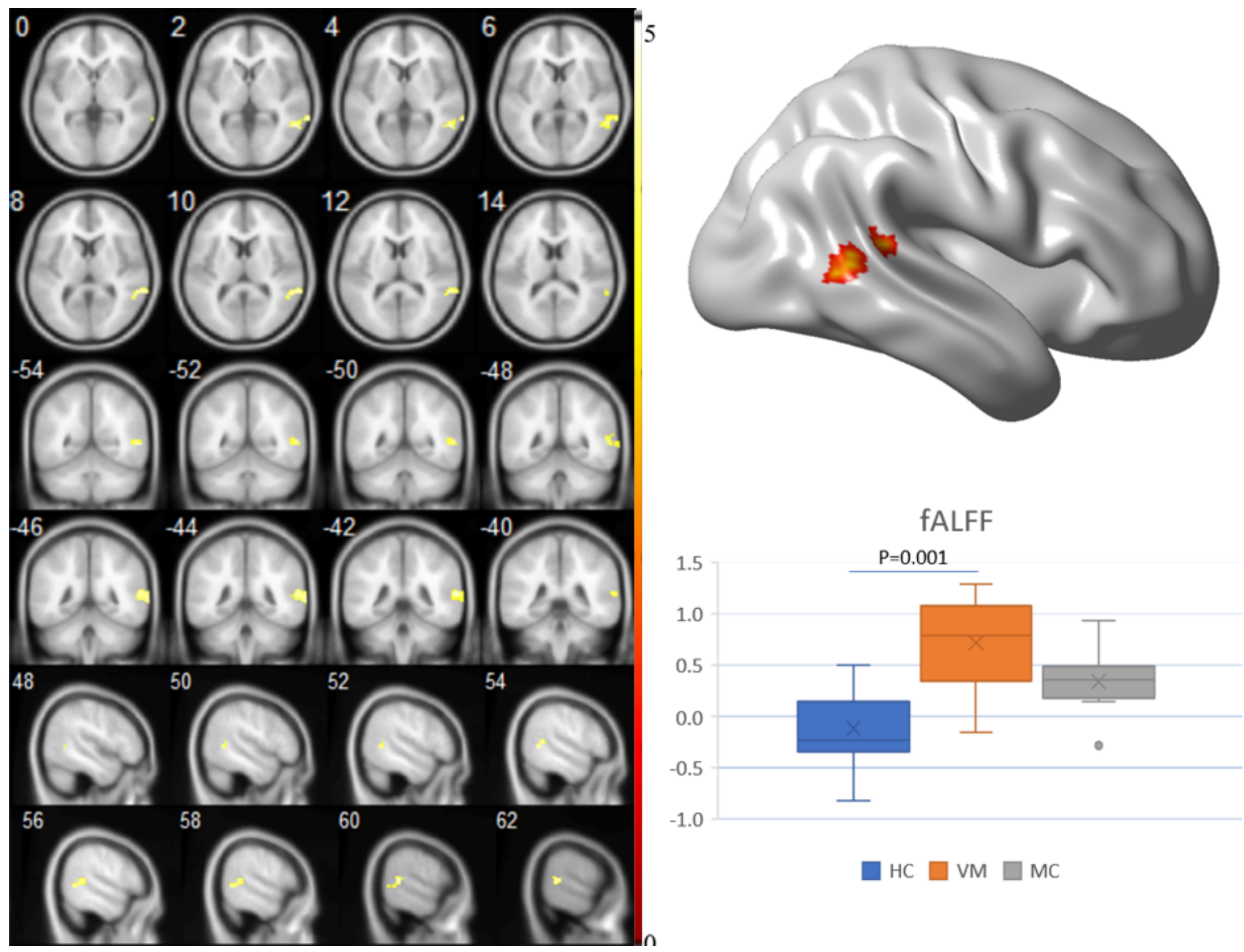

Figure 2

Differences in fALFF values between three groups. Vestibular migraine $(\mathrm{VM})$ group had significantly increased fALFF values in the right temporal lobe compared with health control $(\mathrm{HC})$ group $(X=69, Y=-45, Z=3)$, indicating that spontaneous functional activity in this area was enhanced in patients with VM, which corroborated the ALFF results. No significant difference was found between migraine and HC groups, as well as VM and migraine groups. 

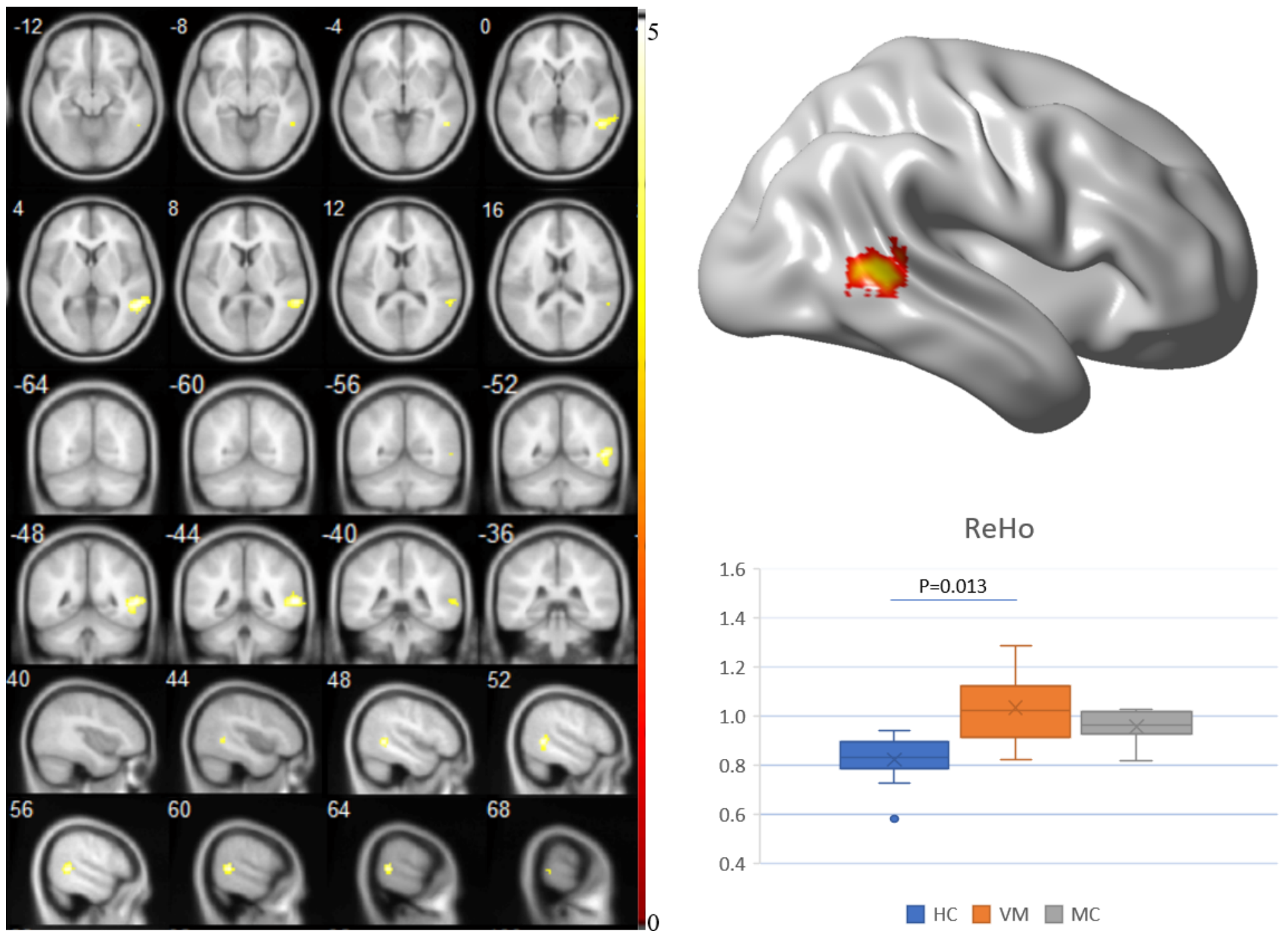

Figure 3

Differences in ReHo values between the three groups. Vestibular migraine (VM) group showed significantly increased ReHo values in the right temporal lobe, including superior temporal gyrus (STG) and middle temporal gyrus (MTG) and inferior temporal gyrus (ITG), compared with health control (HC) group $(X=60, Y=-45, Z=6, P=0.013$, FWE corrected). No significant difference was found between migraine and $\mathrm{HC}$ groups, as well as VM and migraine groups. 

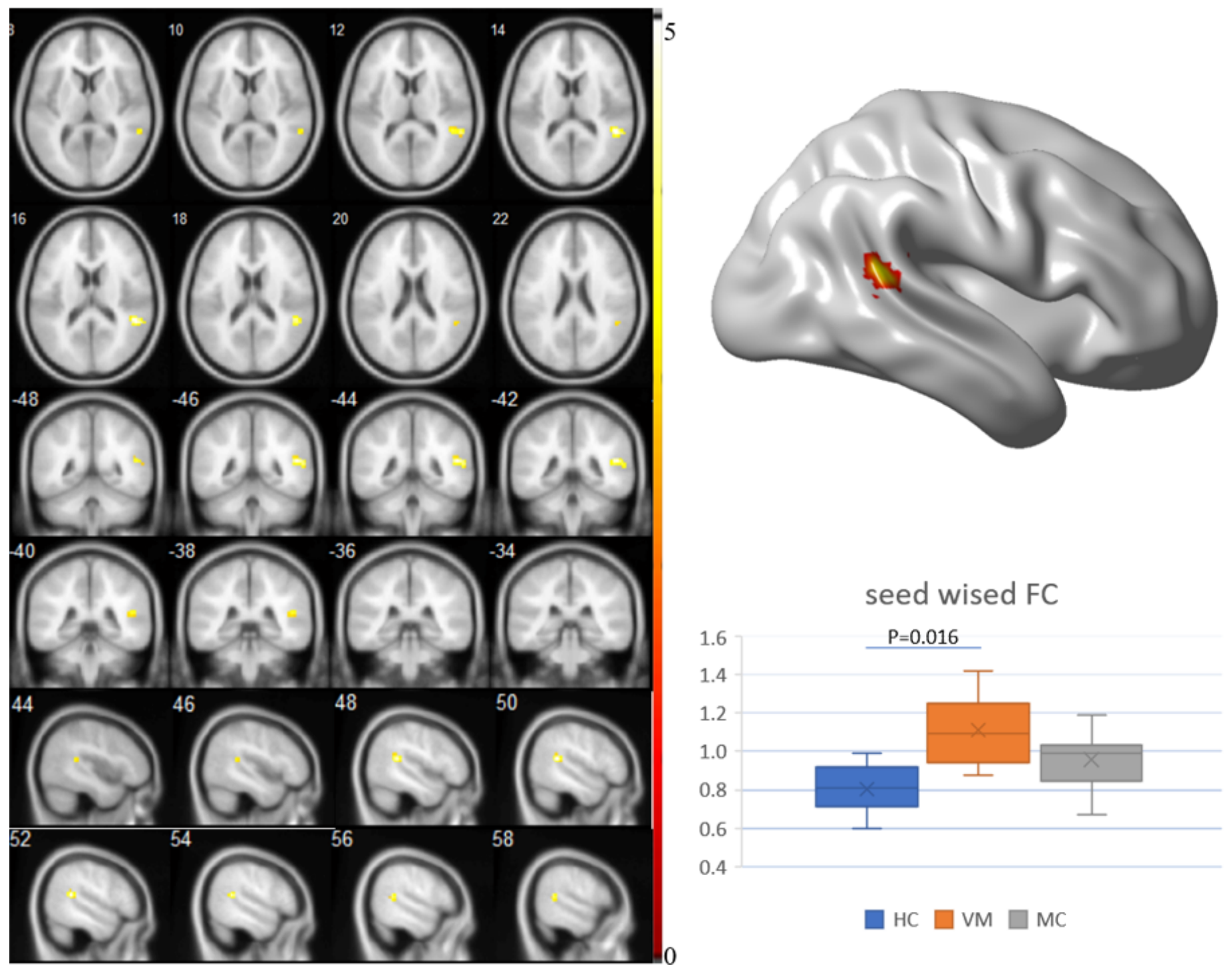

Figure 4

Differences in functional connectivity (FC) between the three groups. Vestibular migraine (VM) group showed significantly increased FC in the right temporal lobe $(X=51, Y=-45, Z=15)$ compared with health control $(\mathrm{HC})$ group, indicating that functional connectivity between this region and the seed regions was enhanced $(X=69, Y=-45, Z=3)$, which corroborated the ReHo results. No significant difference was found between migraine and $\mathrm{HC}$ groups, as well as VM and migraine groups.

\section{Supplementary Files}

This is a list of supplementary files associated with this preprint. Click to download.

- 1657317173667707BIBChecklist.docx 